\title{
BMJ Open Changes in paediatric dental clinic after reopening during COVID-19 pandemic in Wuhan: a retrospective study
}

\author{
Jinghui Yang (D) , ${ }^{1,2}$ Guobin Yang, ${ }^{1,3}$ Runze Jin, ${ }^{1,2}$ Guangtai Song (D) , ${ }^{1,2}$ \\ Guohua Yuan ${ }^{1,2}$
}

To cite: Yang J, Yang G, Jin R, et al. Changes in paediatric dental clinic after reopening during COVID-19 pandemic in Wuhan: a retrospective study. BMJ Open 2022;12:e048430. doi:10.1136/ bmjopen-2020-048430

- Prepublication history and additional supplemental material for this paper are available online. To view these files, please visit the journal online (http://dx.doi.org/10.1136/ bmjopen-2020-048430).

Received 27 December 2020 Accepted 25 September 2021

Check for updates

(C) Author(s) (or their employer(s)) 2022. Re-use permitted under CC BY-NC. No commercial re-use. See rights and permissions. Published by BMJ.

${ }^{1}$ The State Key Laboratory Breeding Base of Basic Science of Stomatology (Hubei-MOST)

and Key Laboratory of Oral

Biomedicine Ministry of

Education, Wuhan University,

Wuhan, Hubei, China

${ }^{2}$ Department of Paediatric

Dentistry, School and Hospital of Stomatology, Wuhan University,

Wuhan, Hubei, China

${ }^{3}$ Department of General and Emergency, School and Hospital of Stomatology, Wuhan University, Wuhan, Hubei, China

Correspondence to

Professor Guohua Yuan; yuanguohua@whu.edu.cn

\section{ABSTRACT}

Objectives Affected by COVID-19 pandemic, The Department of Paediatric Dentistry of School and Hospital of Stomatology, Wuhan University was closed in late January 2020, and resumed on 20 April. Our study aimed to explore the effects of COVID-19 pandemic on paediatric dental services which might assist global paediatric dentists to build confidence and make appropriate policies under the pandemic.

Design A retrospective study was performed. Medical records of patients were retrieved but without any private information, including patient name, ID number and address.

Participants All data of the patients from 20 April to 31 July in 2020 and 2019 were extracted and analysed including demographics, dental diagnosis and treatment methods. A total of 18198 patients were included in the study.

Results During this period, no medical staff or patient was infected with COVID-19 due to dental services. A total of 6485 in 2020 but 11713 during the same period in 2019 visited the department. Compared with 2019, gender distribution did not change, but age distribution changed with an increase under 6 -year-old. The diagnoses including caries, retained primary teeth, malocclusion, deep pits and fissures changed significantly, while pulpitis, apical periodontitis, tooth trauma, early loss of primary teeth, supernumerary teeth showed little change. Aerosol generating procedures were adopted less frequently overall in this period.

Conclusions The reopening of paediatric dental services is proceeding steadily with significant changes in the characteristics of the patients and treatment procedures.

\section{INTRODUCTION}

In December 2019, COVID-19 emerged in Wuhan and spread rapidly across the globe, becoming a major public health challenge for countries around the world. ${ }^{1}$ The COVID-19 pandemic led to a high case fatality rate up to $2.12 \%$ in WHO region-wise till February $2021 .^{23}$ A novel highly infectious corona RNA virus (named SARS-CoV-2) was responsible for the COVID-19, which could transmit via droplets or direct contact with infected people or contaminated surfaces. ${ }^{4}$ Due to the transmission

\section{Strengths and limitations of this study}

- Based on large sample size, the study explored the influence caused by COVID-19 pandemic on paediatric dentistry.

- Our measures and experience to prevent and control the COVID-19 transmission at the paediatric dental clinic were shared.

- The short-term impact of COVID-19 on paediatric dental care in Wuhan was explored.

- The study only analysed the situation in our hospital, which might not be applicable to some countries or areas.

- Due to the limited time, further research is needed to discuss the medium-term and long-term impact of the COVID-19 on paediatric dental clinic.

routes of COVID-19, many high-contact professions including dentistry were severely affected. ${ }^{5}$ Dental healthcare professionals worked in close proximity to patients. And most dental procedures were aerosol generating procedures (AGPs) which caused a large number of droplets and aerosols, such as use of a high-speed or low-speed handpieces, ultrasonic instruments and water-air syringes. ${ }^{6-9}$ The standard protective measures were not sufficient to prevent the spread of COVID-19 because of the unique characteristics of dentistry, especially when patients were in the incubation period, or unaware they had been infected. ${ }^{7}$ Therefore, among healthcare personnel, dentists were at an elevated risk of exposure to COVID-19. This risk was even more serious in paediatric dentistry, because affected children frequently presented asymptomatic, mild or moderate clinical manifestations. ${ }^{10-12}$ To prevent and control transmission of COVID-19, Wuhan city was locked down on 23 January 2020. On the second day, Hubei province activated level 1 response to public health emergencies. Then all elective treatments to dental patients in Wuhan city were postponed and only emergency dental care was reserved. ${ }^{1314}$ 
The Department of Paediatric Dentistry in WHUSS (School and Hospital of Stomatology, Wuhan University) was the main centre for the routine treatment and care for children's dental diseases in Wuhan. This department provided dental care to around 36000 children in 2019. From 24 January to 19 April 2020, clinical procedures of this department were completely stopped. During this period, online consultations and oral health instructions were provided, and severe dental emergencies were recommended to the Department of General and Emergency in WHUSS, which was the only available department in WHUSS special for emergency dental services. ${ }^{14}$ Although it was important to provide treatment for children who presented with urgent or emergency dental complaints, dental non-emergencies that failed to receive immediate intervention were at an increased risk to exacerbate and even developed urgent or emergent complications. Therefore, the contradiction between the huge paediatric demands and the limited dental service worsened after the closure of the Department of Paediatric Dentistry. ${ }^{6913}$ After being imposed a lockdown for as long as 76 days, Wuhan city successfully contained the spread of the virus. On 8 April 2020, all travel restrictions in and out of Wuhan was removed. ${ }^{15}$ On 20 April, the Paediatric Department of WHUSS was reopened and aimed to improve children's dental healthcare.

Similar to Wuhan in China, paediatric dental treatments were completely stopped or significantly influenced in several affected countries. ${ }^{6} 16-18$ Paediatric dentistry was essential children's healthcare. During this evolving and unprecedented COVID-19 pandemic period, it was an important issue to get a balance between prevention of potential exposure to SARS-CoV-2 infection and minimisation of the harm to patients from postponed dental care. ${ }^{1618}$ New protocols and measures were needed as dental professionals returned to normal practice after weeks or months of confinement in many countries. Some articles have discussed the management of dental practice during the COVID-19 pandemic, ${ }^{356}$ but few articles explored the situation and changes after the recovery of dental clinics based on large sample data.

In this study, we collected the data of the patients from 20 April to 31 July in 2020 and the corresponding period in 2019 to analyse the impact of the COVID-19 pandemic on the children's dental care. We also shared our experience on restructuring the patient pathway and workflow of healthcare personnel.

\section{MATERIALS AND METHODS Inclusion and exclusion criteria}

The information of the patients who visited the Department of Paediatric Dentistry, WHUSS from 20 April to 31 July in 2020 and the corresponding period in 2019 was retrieved and compared (all private information has not been extracted, including patient name, ID number and address). Any medical record had one or even more information missing was excluded.

\section{Patient and public involvement}

Reporting of the study follows the Strengthening the Reporting of Observational Studies in Epidemiology guidelines. Patients or the public were not involved in the design, or conduct, or reporting, or dissemination plans of our research

\section{Workflow of the dental practice personnel}

In order to ensure safety after reopening of the hospital, a series of measures had been applied among medical staff which mainly included: nucleic acid testing for all before reopening, personal protection training for employees, adequate provision of personal protective equipment and a complete clinic management system. Please refer to the online supplemental materials for details.

\section{Patient pathway for paediatric dental services}

During the pandemic, WHUSS and p;aediatric dentistry formulated protocols to prevent nosocomial infections of COVID-19. These measures included: online appointment system, medical history collection of COVID-19, body temperature monitoring, provision of nucleic acid reports and so on. Patients needed to make an appointment online in advance, those who lacked an appointment would not be allowed to enter the hospital. The patient triage based on the COVID-19 exposure history was completed on patient arrival. Children and their accompanying persons were required to provide the appointment messages, the children's identity information and the accompanying person's health QR-code. All people needed to receive body temperature assessment. Please refer to the online supplemental materials for details. The questionnaires for COVID-19 are shown in online supplemental appendix tables 1 and 2.

\section{Data collection and management}

Data collection included number and demographic information of visited children (age and gender), their diagnosis and treatment options provided by dentists. Data associated with COVID-19 were obtained from the government. ${ }^{19}$

All data were classified according to specific standards. Age was divided into four groups: 0-3 years old, 4-6 years old, 7-12 years old, 13 years old and above. For the diagnosis of children, nine common diseases were listed while some uncommon diseases such as traumatic ulcer, central cusp, gingivitis were grouped into other. Treatments were divided into Oral Health Consultation group (they received a comprehensive oral examination and oral health instruction without any dental procedures.) and Oral Health Consultation \& Treatment group. The latter group was further divided into AGPs Treatment Group and non-AGPs Treatment Group according to whether any AGPs was used.

\section{Figure drawing and statistical analysis}

The data were entered into Excel 2019 (Microsoft corporation, Redmon, Washington, USA) and imported into SPSS V.25.0 (IBM Corporation) for statistical analysis. 


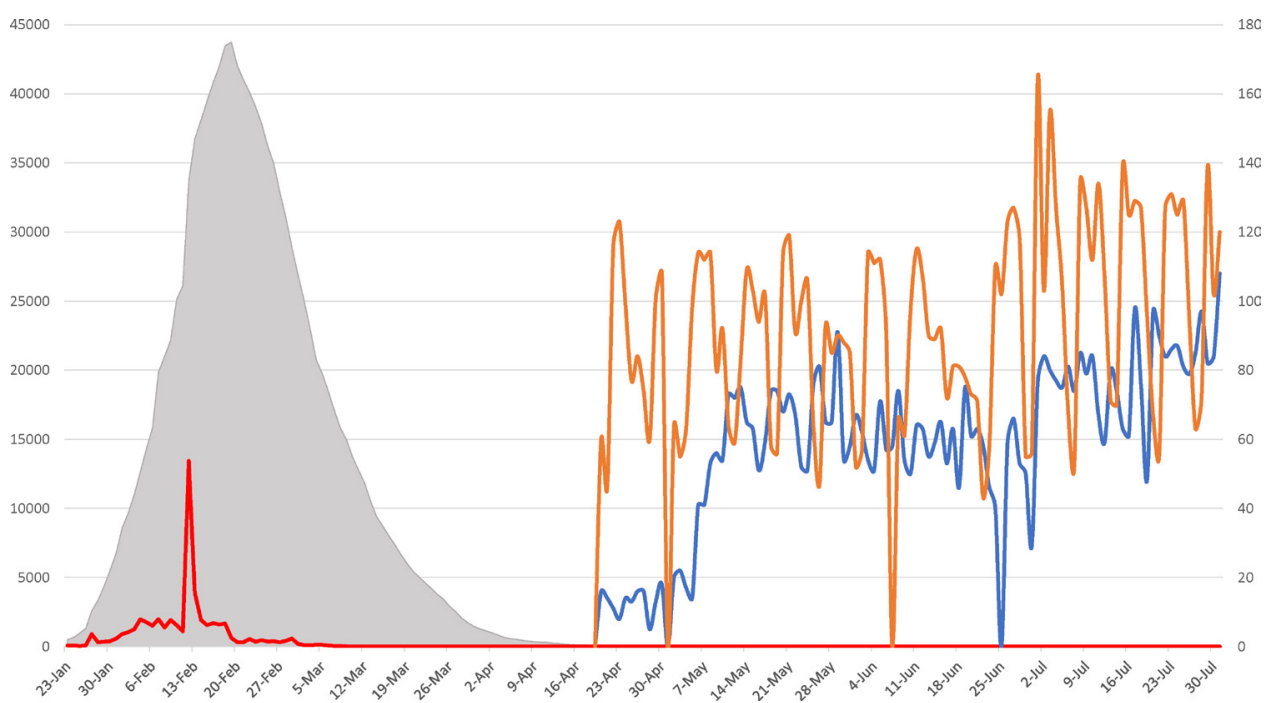

Figure 1 The situation of COVID-19 pandemic in Wuhan, Hubei, and daily visits at the Department of Paediatric Dentistry in WHUSS from 20 April to 31 July in 2020 and 2019. The grey shading shows the number of patients with hospitalised COVID-19 in Hubei Province. The red line shows the number of new confirmed COVID-19 cases in Wuhan city. The blue and orange lines represent the number of patients in 2020 and 2019, respectively.

Figures were drawn by Excel. Pearson $\chi^{2}$ test was used to verify the difference of categorical variables. Significance levels were set as $\mathrm{p}<0.05$ using any test.

\section{RESULTS}

The overall recovery process of the Department of Paediatric Dentistry in WHUSS

From 20 April to 31 July, a total of two medical staff experienced fever symptoms. Their test reports on nucleic acid of SARS-CoV-2 were both negative and were diagnosed as influenza instead of COVID-19. As of 31 July, no medical staff or patient had contracted COVID-19 due to medical services.

After reopening on 20 April, a series of measures had been taken to prevent COVID-19 infections and ensure the safety of medical staff and patients. These measures included: changes in the appointment system from a combination of on-site and online to online only; strict verification of identification information before entering the hospital and clinic area; collection of epidemiological information related to COVID-19 of each patient and perfect classified diagnosis and treatment system and so on. All the above measures (Methods and online supplemental materials) ensured the recovery process of the Department of Paediatric Dentistry of WHUSS to be promoted steadily.

The trend of COVID-19 pandemic in Wuhan (23 January to 31 July 2020) is shown in figure 1 . The number of daily visits from the reopening day to 31 July 2020 (the blue line) and during the corresponding period in 2019 (the orange line) were retrieved. The daily visits in 2019 were at a comparably stable level, about 80 for average. In 2020, the number of visits remained very low in the early recovery stage (late April), and gradually increased. In the early first half of May, the average daily visits reached nearly 50 , returning to $60 \%$ of the same period last year. Since late May, the number of the patients reached a relatively stable level, reaching 60 , which was around $60 \%-80 \%$ of the same period in 2019.

\section{Changes in the basic information of the children visiting WHUSS}

To further understand the possible changes of the patients' characteristics, basic information of the children who visited the Department of Paediatric Dentistry in WHUSS was obtained. Table 1 shows their gender and age distribution. No significant difference was found in the gender distribution between 2019 and 2020. But a significant difference was seen in the age distribution with an increase of the children under 6 years old (0-3 and 4-6 years old groups) but a decrease of the elder children in 2020.

\section{Changes in the diagnoses of the children visiting WHUSS}

The disease diagnoses of the visited children in 2020 are shown in table 2. Since the absolute number of children visiting varied greatly between 2020 and 2019, the percentages of the number of each diagnosis were further compared. Comparison among the 4 months within 2020 , the diagnosis $(\%)$ of retained primary teeth declined month by month. However, compared with the total patients in 2019, the COVID-19 pandemic has led to a significant increase of the percentage of retained primary teeth. Meanwhile, significant impacts of COVID-19 on the percentage of dental caries, malocclusion, deep pits and fissures were also seen, with an increase in the first diagnosis but decreases in the latter two. The detailed diagnoses for patients in 2019 are shown in online supplemental appendix table 3 . 
Table 1 Gender and age distribution of the children who visited the Department of Paediatric Dentistry in WHUSS from 20 April and 31 July in 2019 and 2020

\section{$2020(n=6485) 2019(n=11713)$}

(\%)

(\%)

\begin{tabular}{lcc}
\hline $\begin{array}{l}\text { Gender } \dagger \\
\text { Male }\end{array}$ & $3429(52.88)$ & $6280(53.62)$ \\
Female & $3056(47.12)$ & $5433(46.38)$ \\
Age ‡ $^{*}$ & \\
0-3 years old & $718(11.07)$ & $593(5.06)$ \\
4-6 years old & $2745(42.33)$ & $4152(35.45)$ \\
7-12 years old & $2839(43.78)$ & $6229(53.18)$ \\
13 years old and above & $183(2.82)$ & $739(6.31)$ \\
\hline
\end{tabular}

The symbol * represents significant difference using Pearson $\chi^{2}$ test $(\mathrm{P}<0.05)$.

†There is no significant difference of the gender of the children between 2019 and 2020 using Pearson $\chi^{2}$ test $\left(\chi^{2}=0.918\right.$,

$\mathrm{p}=0.338>0.05$ )

$\ddagger$ The age distribution between 2019 and 2020 was significantly different with an obvious increase of the children under 6 years old (0-3 and 4-6 years old group) and a decrease of the children above 7 years old in 2020 using Pearson $\chi^{2}$ test $\chi^{2}=435.590$, $\mathrm{p}<0.001$ ).

WHUSS, School and Hospital of Stomatology, Wuhan University.

\section{Changes in the treatment operations}

In order to analyse the impact of COVID-19 on the implement of dental treatment operations, the data were further collected and shown in table 3. On the whole, a higher proportion of children left the hospital after receiving oral health consultations only in 2020. But from April to July, the proportion of children undergoing treatment operations was increasing while in 2019, the ratio between these two groups was stable. By comparing
Table 3 All visits were grouped into Oral Health Consultation group and Oral Health Consultation \& Treatment group according to whether treatment operation(s) was adopted

\begin{tabular}{|c|c|c|c|c|}
\hline Time $\dagger^{\star} \ddagger \S^{\star}$ & $\begin{array}{l}\text { Total } \\
\text { visits }\end{array}$ & $\begin{array}{l}\text { Oral Health } \\
\text { Consultation } \\
\text { only (\%) }\end{array}$ & $\begin{array}{l}\text { Oral Health } \\
\text { Consultation } \\
\text { \& Treatment } \\
(\%)\end{array}$ & $P$ value \\
\hline April 2020 & 159 & $81(50.94)$ & 78 (49.06) & $\mathrm{p}<0.001^{*}$ \\
\hline April 2019 & 1129 & 332 (30.92) & 797 (69.08) & \\
\hline May 2020 & 1879 & $581(34.46)$ & $1298(65.54)$ & $p=0.006^{\star}$ \\
\hline May 2019 & 3205 & $876(27.48)$ & 2329 (72.52) & \\
\hline June 2020 & 1805 & $622(29.41)$ & 1183 (70.59) & $\mathrm{p}<0.001^{\star}$ \\
\hline June 2019 & 3111 & 898 (27.33) & $2213(72.67)$ & \\
\hline July 2020 & 2642 & $726(28.87)$ & 1916 (71.13) & $p=0.511$ \\
\hline July 2019 & 4268 & $1204(28.21)$ & 3064 (71.79) & \\
\hline
\end{tabular}

The symbol * represents significant difference using Pearson $\chi^{2}$ test $(\mathrm{P}<0.05)$.

†A significant difference was seen among the 4 months within 2020 (Pearson $\chi^{2}$ test, $\chi^{2}=54.989, p<0.001$ ).

$\ddagger$ There is no significant difference among the 4 months within 2019 (Pearson $\chi^{2}$ test, $\chi^{2}=2.660, p=0.447>0.05$ ).

$\S$ There is a significant difference between the total amount (20 Apr to 31 July) of each group in 2019 and 2020 using Pearson $\chi^{2}$ test $\left(\chi^{2}=15.097, p<0.001\right)$.

the same month of 2019 and 2020, it was found that there were significant differences in April, May and June, but there was no significant difference in July.

Considering the high risks of AGPs for COVID-19 transmission, the Oral Health Consultation \& Treatment group was classified into two groups according to whether any AGPs was used (table 4). Comparison between 2019 and 2020 showed that the proportion of AGPs operations

Table 2 The changes in the patients' diagnoses from 20 April and 31 July in 2020. Nine common diagnoses were listed in the table. Other uncommon diagnoses were classified as 'Other'

\begin{tabular}{|c|c|c|c|c|c|c|}
\hline & Apr $2020(\%)$ & May $2020(\%)$ & Jun 2020 (\%) & Jul 2020 (\%) & Total in $2020(\%)$ & Total in $2019(\%)$ \\
\hline Caries $\dagger \ddagger^{*}$ & $49(30.82)$ & $586(31.19)$ & 577 (31.97) & 836 (31.64) & 2048 (31.58) & 3388 (28.93) \\
\hline Pulpitis $† \ddagger$ & $8(5.03)$ & $151(8.04)$ & $171(9.47)$ & $257(9.73)$ & $587(9.05)$ & $1144(9.77)$ \\
\hline Retained primary teeth $\dagger^{\star} \ddagger^{*}$ & $30(18.87)$ & $286(15.22)$ & $184(10.19)$ & $236(8.93)$ & 736 (11.35) & $1010(8.62)$ \\
\hline Malocclusion $\dagger \ddagger^{*}$ & $13(8.18)$ & $159(8.46)$ & $145(8.03)$ & $229(8.66)$ & $546(8.42)$ & $1128(9.63)$ \\
\hline Deep pits and fissures $\dagger \ddagger^{*}$ & $3(1.89)$ & $26(1.38)$ & $28(1.55)$ & $45(1.70)$ & $102(1.57)$ & $255(2.18)$ \\
\hline Supernumerary teeth $† \ddagger$ & $4(2.52)$ & $57(3.03)$ & $47(2.60)$ & $72(2.73)$ & $180(2.78)$ & $312(2.66)$ \\
\hline Other $\dagger^{*} \ddagger$ & $6(3.77)$ & $82(4.36)$ & $129(7.15)$ & $193(7.31)$ & $410(6.32)$ & $823(7.03)$ \\
\hline
\end{tabular}

The monthly diagnoses in 2019 were shown in online supplemental materials.

The symbol * represents significant difference using Pearson $\chi^{2}$ test $(P<0.05)$.

†Comparison among the 4 months within 2020.

¥Comparison of the patients' diagnoses between the total period in 2020 and 2019 . 
Table 4 The patients treated by dental procedures were classified into two groups according to whether any AGPs was used

\begin{tabular}{|c|c|c|c|c|}
\hline Time $\dagger^{*} \ddagger \S^{\star}$ & $\begin{array}{l}\text { Treated } \\
\text { patients }\end{array}$ & $\begin{array}{l}\text { AGPs } \\
\text { treatment (\%) }\end{array}$ & $\begin{array}{l}\text { Non-AGPs } \\
\text { treatment (\%) }\end{array}$ & $P$ value \\
\hline Apr 2020 & 78 & $10(12.82)$ & $68(87.18)$ & $p<0.001^{*}$ \\
\hline Apr & 797 & 504 (63.23) & $6.76)$ & \\
\hline May 2020 & 1298 & $383(29.51)$ & 915 (70.49) & $\mathrm{p}<0.001^{*}$ \\
\hline May 2019 & 2329 & 1387 (59.55) & $942(40.45)$ & \\
\hline June 2020 & 1183 & $603(50.97)$ & $580(49.03)$ & $p<0.001^{*}$ \\
\hline June 2019 & 2213 & $1310(59.20)$ & $903(40.80)$ & \\
\hline July 2020 & 1916 & $1010(52.71)$ & 906 (47.29) & $\mathrm{p}<0.001^{*}$ \\
\hline July 2019 & 3064 & $1883(61.46)$ & $1181(38.54)$ & \\
\hline
\end{tabular}

The symbol * represents significant difference using Pearson $\chi^{2}$ test $(\mathrm{P}<0.05)$.

†A significant change was seen among the 4 months within 2020 with an obvious monthly increase (Pearson $\chi^{2}$ test, $\chi^{2}=221.737, p<0.001$ ). $\ddagger$ There is no significant difference among the 4 months within 2019 (Pearson $\chi^{2}$ test, $\chi^{2}=6.118, p=0.106$ ).

$\S$ There is a significant difference between the total amount $(20 \mathrm{Apr}$ to 31 July) of each group in 2019 and 2020 using Pearson $\chi^{2}$ test $\left(\chi^{2}=289.960, p<0.001\right)$.

AGP, aerosol generating procedure.

dropped and non-AGPs treatment increased in 2020. Among April to July in 2020, the proportion of AGPs treatment showed an obvious increase month by month, but still at a lower level than the corresponding month of 2019. In April 2020, only 10 cases were operated with AGPs. Gradually, such attempts had been expanded, reaching nearly $30 \%$ in May, which increased month by month, finally reaching a level similar to 2019 in July.

Paediatric dental clinic provides all oral healthcare for children and adolescents which includes extensive procedures. Some representative treatment methods were selected and percentages of these treatment methods were calculated. The average number of teeth treated during a single visit was also displayed in online supplemental figure 1 .

\section{DISCUSSIONS}

After Wuhan city experienced the COVID-19 pandemic attack in 2020, the medical industry including dental care service had been seriously affected. To explore the changes of paediatric dental services during the pandemic, the information of children who visited the Department of Paediatric Dentistry of WHUSS including gender, age, diagnoses and treatment options they received was extracted. By comparing with the same period in 2019, we found that the COVID-19 pandemic had an impact on the policies of dental hospitals and clinics, the distribution of children's age and oral diseases, as well as the treatment operations to the patients.

The strategy of pathway for patients and the performance of patient triage were reorganised and adopted till now. However, it still had some limitations. The collection of COVID-19 exposure history as well as the checking for body temperature were completed only on patient arrival. ${ }^{20}{ }^{21}$ But if the patient or the accompany person was a potential COVID-19 infected person or had been contacted with an infected person, the spread of COVID-19 was hard to be avoided on the way to the hospital. Judson et al proposed self-triage and selfscheduling based on the phone, which was being rapidly adopted on health systems around the world. ${ }^{22}$ So this kind of system for patient triage, both on the phone and on patient arrival, which had the potential to greatly improve triage efficiency and prevent unnecessary visits during the COVID-19 pandemic, was a valuable measure for risk assessment of COVID-19 for patients. ${ }^{22} 23$

The regular dental healthcare in Wuhan was influenced by COVID-19 pandemic, including paediatric patients and dentists. The COVID-19 pandemic had affected children's dental healthcare in Wuhan, and the short-term changes in children caused by COVID-19 pandemic were mainly due to community closure and delayed treatment. A significant increase was observed in the children at younger ages ( $0-6$ years old), which might be attributed to the closure of the community. During this period, children were required to stay at home all day, feeling upset by losing outdoor activities, and daily dental care was sometimes ignored. ${ }^{24}$ Coupled with poor self-control and poor oral cleaning, young children were more prone to dental diseases. At the same time, due to the closure, children were unable to visit the hospital to receive regular oral examination and the fluoride anti-caries treatment, which made the incidence of dental caries further increased. ${ }^{25}$

At present, the impact of delayed treatment on children mainly lead to the change of the reasons for these children's visit, which was mainly reflected in the change of disease distribution in our study. A significant decrease for dental trauma was found in 2020 (April to June), which may be attributed to reduced outdoor activities. Although community closure was cancelled at the end of March, educational institutions especially kindergartens and elementary schools did not return to work at that time. To avoid the possibility of infection, most children were required to go out less frequently by their caregivers. ${ }^{26}$ In Wuhan, China, summer vacation usually started from early July. It was found that for 2019, the percentage of dental trauma in July also reduced greatly either.

Hopcraft and Farmer investigated the impact of COVID-19 on dental services in Australia from February 2020 to September, finding that the pandemic had a significant impact on the provision of dental services to children from lower socioeconomic backgrounds, and the delayed dental care was likely to contribute to poorer oral health and long-term problems for many Australians. ${ }^{27}$ Different from this study, we found that the impact of the COVID-19 pandemic on Wuhan mainly concentrated in the first 3 months after reopening. In the fourth month (July 2020), the characteristics of children gradually returned to normal. It was speculated that the restriction measures in Wuhan lasted only 3 months. 
If the restrict time was prolonged, the impact might be more serious. Therefore, our study found that the impact of COVID-19 pandemic and the deferral of dental treatment in Wuhan mainly focused on the short-term impact, while the medium-term and long-term impact might be limited, needing more research to explore.

Another obvious change occurred in the dentists' treatment concepts, breaking the original routines. ${ }^{5-7}$ Especially in late April when the outpatient service just recovered, more than half of the children did not receive any operation in the initial appointment. In addition, some parents or caregivers were also worried about the risk of COVID-19 infection by AGPs, so they refused to receive any treatment for their children but dental health consultation. With the resumption process proceeding in an orderly manner, the concerns of both dentists and parents decreased, so more dentists and parents were willing to receive AGPs till the end of July.

Many current guidelines recommend minimising the use of AGPs, ${ }^{7928} 29$ but non-AGPs operations cannot solve some common dental diseases, such as endodontic treatments for pulpitis. Taking into account the complexity of children's oral diseases and their limited ability of oral hygiene control, failing to intervene in time will worsen the diseases and eventually make more complicated treatments necessary. Nevertheless, since the global pandemic has not yet been eliminated, the possibility of a recurrence of COVID-19 in Wuhan cannot be ruled out. Some medical staff hold the view that preventive measures must also be paid attention to such as fluoride anti-caries treatment. ${ }^{30}$ The application of fluoride can effectively prevent dental caries, which will reduce the probability of these children suffering from dental caries in the future and reduce their visits to the hospital.

As a retrospective report, we extracted and classified the data of the 4 months since the reopening of dental healthcare work in the Paediatric Dentistry of WHUSS. In-depth comparisons were conducted from three aspects, namely: comparison within 2019, comparison within 2020 and comparison between 2019 and 2020. In 2019, the data were mostly stable, and the dentists' treatment choice was directly determined by the patient's disease diagnosis, with no extra factors to worry about. The only fluctuation came from the summer vacation in July as discussed above. However, in 2020, a large amount of changes happened. When dentists just returned to work on April, most of them tried to avoid AGPs operations while parents also refused to take their children to hospital when it was not urgent enough regarding the danger of hospital environment. In this exploratory period, the attitudes of dentists and patients were relatively conservative. At the end of May, Wuhan city carried out nucleic acid tests on all citizens, which gave both dentists and patients incentives. The numbers of dentists at work, visited patients, patients treated and patients receiving AGPs also increased. This phenomenon was more significant from May to June. When entering July, after the first 3 months of exploration, the concepts of both dentists and patients have become stable. This month-to-month change has begun to shrink, approaching the degree compared with the same month in 2019. The overall reopening process has achieved phased success.

\section{CONCLUSION}

The COVID-19 pandemic has indeed brought an impact on children's dental care. Under this attack, lower visit number as well as younger age distribution can be found. And AGPs operations were used less frequently. Our department resumed medical service at the end of April, without any example to follow. Through the efforts of various departments of the hospital, plans and medical procedures have been formulated. Looking back at our reopening process, it cannot be perfect enough, but it is still satisfactory. Our experience may help global paediatric dental healthcare workers build confidence and make appropriate policies for resuming high-quality dental services during COVID-19 pandemic.

Contributors JY contributed to design, data acquisition and analysis, drafted manuscript and critically revised manuscript. GY contributed to design, interpretation, drafted manuscript and critically revised manuscript. RJ contributed to design, drafted manuscript and critically revised manuscript. GS contributed to conception, data acquisition and critically revised manuscript. GY contributed to conception and design, analysis and interpretation, drafted manuscript and critically revised manuscript, and is responsible for the overall content as guarantor. All authors gave their final approval and agreement to be accountable for all aspects of the work.

Funding This research was supported by grants from the National Natural Science Foundation of China (Number: 82170914).

\section{Competing interests None declared.}

Patient consent for publication Not applicable.

Ethics approval This study involves human participants and was approved by the Ethics Committee of School and Hospital of Stomatology, Wuhan University with the reference ID No. 2020-B70. Consent for participation in the study was not obtained because this retrospective study did not interfere with patients, nor did it involve any patient privacy. And due to the large sample size, not all patients could be found so consent for participation was not obtained. This study does not involve animal subjects.

Provenance and peer review Not commissioned; externally peer reviewed.

Data availability statement Data are available upon reasonable request. Data including detailed medical record without any private information is available upon reasonable request.

Supplemental material This content has been supplied by the author(s). It has not been vetted by BMJ Publishing Group Limited (BMJ) and may not have been peer-reviewed. Any opinions or recommendations discussed are solely those of the author(s) and are not endorsed by BMJ. BMJ disclaims all liability and responsibility arising from any reliance placed on the content. Where the content includes any translated material, BMJ does not warrant the accuracy and reliability of the translations (including but not limited to local regulations, clinical guidelines, terminology, drug names and drug dosages), and is not responsible for any error and/or omissions arising from translation and adaptation or otherwise.

Open access This is an open access article distributed in accordance with the Creative Commons Attribution Non Commercial (CC BY-NC 4.0) license, which permits others to distribute, remix, adapt, build upon this work non-commercially, and license their derivative works on different terms, provided the original work is properly cited, appropriate credit is given, any changes made indicated, and the use is non-commercial. See: http://creativecommons.org/licenses/by-nc/4.0/.

\section{ORCID iDs}

Jinghui Yang http://orcid.org/0000-0002-6468-083X

Guangtai Song http://orcid.org/0000-0002-5416-1665 


\section{REFERENCES}

1 Zhu N, Zhang D, Wang W, et al. A novel coronavirus from patients with pneumonia in China, 2019. N Engl J Med 2020;382:727-33.

2 Fu L, Wang B, Yuan T, et al. Clinical characteristics of coronavirus disease 2019 (COVID-19) in China: a systematic review and metaanalysis. J Infect 2020;80:656-65.

3 Kumar A, Singh R, Kaur J, et al. Wuhan to world: the COVID-19 pandemic. Front Cell Infect Microbiol 2021;11:596201.

4 BaniHani A, Gardener C, Raggio DP, et al. Could COVID-19 change the way we manage caries in primary teeth? Current implications on paediatric dentistry. Int J Paediatr Dent 2020;30:523-5.

5 Jiang Y, Tang T, Mei L. COVID-19 affected patients' utilization of dental care service. Oral Dis 2020 doi:10.1111/odi.13568

6 Al-Halabi M, Salami A, Alnuaimi E, et al. Assessment of paediatric dental guidelines and caries management alternatives in the post COVID-19 period. A critical review and clinical recommendations. Eur Arch Paediatr Dent 2020;21:543-56.

7 Burton MJ, Clarkson JE, Goulao B, et al. Antimicrobial mouthwashes (gargling) and nasal sprays to protect healthcare workers when undertaking aerosol-generating procedures (AGPS) on patients without suspected or confirmed COVID-19 infection. Cochrane Database Syst Rev 2020;9:CD013628.

8 Drum E, McClung Pasqualino H, Subramanyam R. Anesthesia and potential aerosol generation during magnetic resonance imaging in children with COVID-19. Paediatr Anaesth 2020;30:944-6.

9 Mallineni SK, Innes NP, Raggio DP, et al. Coronavirus disease (COVID-19): characteristics in children and considerations for dentists providing their care. Int J Paediatr Dent 2020;30:245-50.

10 Liu T, Liang W, Zhong $\mathrm{H}$, et al. Risk factors associated with COVID-19 infection: a retrospective cohort study based on contacts tracing. Emerg Microbes Infect 2020;9:1546-53.

$11 \mathrm{Su} \mathrm{L}, \mathrm{Ma} \mathrm{X}, \mathrm{Yu} \mathrm{H}$, et al. The different clinical characteristics of corona virus disease cases between children and their families in China - the character of children with COVID-19. Emerg Microbes Infect 2020;9:707-13

12 Yu C, Zhou M, Liu Y, et al. Characteristics of asymptomatic COVID-19 infection and progression: a multicenter, retrospective study. Virulence 2020;11:1006-14.

13 Meng L, Hua F, Bian Z. Coronavirus disease 2019 (COVID-19): emerging and future challenges for dental and oral medicine. J Dent Res 2020;99:481-7. doi:10.1177/0022034520914246

$14 \mathrm{Yu}$ J, Zhang T, Zhao D, et al. Characteristics of endodontic emergencies during coronavirus disease 2019 outbreak in Wuhan. $J$ Endod 2020;46:730-5.

15 Nation Health Commission of the People's Republic of China. The National health Commission held telephone conference to deploy the pandemic prevention after the canceling the restriction for leaving Hubei and Wuhan. Available: http://www.nhc.gov.cn/wjw/xwdt/202004/ 2d61690d515141d2bb584ef1da1050cb.shtml [Accessed 27 Sep 2020].
16 Casamassimo PS, Townsend JA, Litch CS. Pediatric dentistry during and after COVID-19. Pediatr Dent 2020;42:87-90.

17 Ilyas N, Agel M, Mitchell J, et al. COVID-19 pandemic: the first wave - an audit and guidance for paediatric dentistry. Br Dent $J$ 2020;228:927-31.

18 Paglia L. COVID-19 and paediatric dentistry after the lockdown. Eur J Paediatr Dent 2020;21:89.

19 Wuhan Municipal Health Commission. COVID-19 pandemic outbreak notification. Available: http://wjw.wuhan.gov.cn/ztzl_28/fk/yqtb/index. shtml [Accessed 9 Oct 2020].

20 Hsu C-A, Yuan EJ, Fen J-J, et al. Deployment of a computerized ward visitor registration system in coronavirus disease 2019 epidemic: experiences of a large academic medical center in Taiwan. $J$ Chin Med Assoc 2020;83:561-5.

21 Li H, Liu S-M, Yu X-H, et al. Coronavirus disease 2019 (COVID-19): current status and future perspectives. Int J Antimicrob Agents 2020;55:105951.

22 Judson TJ, Odisho AY, Neinstein AB, et al. Rapid design and implementation of an integrated patient self-triage and self-scheduling tool for COVID-19. J Am Med Inform Assoc 2020;27:860-6.

23 Nakamoto I, Wang S, Guo Y, et al. A QR Code-Based contact tracing framework for sustainable containment of COVID-19: evaluation of an approach to assist the return to normal activity. JMIR Mhealth Uhealth 2020;8:e22321.

24 Ahmed HO. The impact of social distancing and self-isolation in the last corona COVID-19 outbreak on the body weight in Sulaimani governorate- Kurdistan/Iraq, a prospective case series study. Ann Med Surg 2020;59:110-7.

25 Yang F, Yu L, Qin D, et al. Online consultation and emergency management in paediatric dentistry during the COVID-19 epidemic in Wuhan: a retrospective study. Int J Paediatr Dent 2021;31:5-11.

26 Yang YT, Zhang W, Xie L. Characteristic changes of traumatic dental injuries in a teaching hospital of Wuhan under transmission control measures during the COVID-19 epidemic. Dent Traumatol 2020.

27 Hopcraft M, Farmer G. Impact of COVID-19 on the provision of paediatric dental care: analysis of the Australian child dental benefits schedule. Community Dent Oral Epidemiol 2021;49:369-76.

28 Khalil MM, Alam MM, Arefin MK, et al. Role of personal protective measures in prevention of COVID-19 spread among physicians in Bangladesh: a multicenter cross-sectional comparative study. SN Compr Clin Med 2020:1733-9.

29 Sobti A, Fathi M, Mokhtar MA, et al. Aerosol generating procedures in trauma and orthopaedics in the era of the Covid-19 pandemic; what do we know? Surgeon 2021;19:e42-8.

30 Spivakovsky S. Which crucial measures do patients need to follow to prevent transmission of COVID-19 in the dental setting? Evid Based Dent 2020;21:79. 